\title{
La ciencia y el conocimiento de la verdad
}

\author{
Evandro Agazzi
}

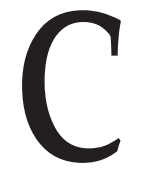

\section{iencia y verdad en la tradición del Occidente}

En la cultura occidental, con el vocablo ciencia constantemente se denotó (hasta el siglo actual) un conocimiento verdadero y aun la forma más perfecta de conocimiento, es decir, un saber dotado de universalidad, necesidad y certeza. Esto resulta evidente si se considera cómo el concepto de ciencia, en el pensamiento griego, resultó de un desarrollo del concepto de verdad. Parménides opone verdad a opinión. Platón reconoce que existen opiniones verdaderas y, dentro del campo de la verdad, distingue la opinión verdadera de la ciencia, la cual es un saber que alcanza las razones de su verdad. Aristóteles confirma y desarrolla esta misma doctrina de la ciencia. En todas estas concepciones el instrumento propio de la verdad es el intelecto, en su doble función de logos que "conecta" las proposiciones y de noûs que es capaz de intuir lo universal de las esencias y de los principios inteligibles. Mientras que en Parménides y Platón el trabajo del intelecto implica un rechazo de la sensación y de los sentidos, en Aristóteles la sensación constituye el punto de partida del conocimiento, ya que el intelecto es capaz de alcanzar por medio de la abstracción las formas inteligibles de las cosas, además de poder intuir los primeros principios totalmente inteligibles del ser. De todos modos, el "lugar" propio de la verdad es el intelecto, en cuanto la verdad es una propiedad del juicio, aunque esta propiedad no resulte de un movimiento puramente "interior" al intelecto, sino del hecho de que éste se conforma a las cosas. En otros términos: la verdad es en el intelecto, pero en virtud de su conformidad con lo real. Esta identidad de pensamiento $y$ ser, afirmada de diversas formas por el pensamiento griego, recibe su esclarecimiento más riguroso en el pensamiento medieval, gracias al desarrollo de la noción de intencionalidad: el intelecto se identifica intencionalmente con las cosas, aunque quedando ontológicamente distinto de ellas. El famoso principio ens 
et verum convertuntur significa simplemente que el verum es el mismo ens en cuanto aprehendido por el intelecto, o sea, en cuanto intencionalmente presente al intelecto.

El pensamiento moderno se desarrolla a partir de un presupuesto dogmático (es decir, que no descansa ni en una evidencia, ni en una argumentación), a saber, que lo que el hombre conoce son sus representaciones (llamadas ideas en un nuevo sentido): éstas, y no las cosas, son "presentes" al pensamiento y por lo tanto se plantea como problema fundamental de la filosofía el de inferir o demostrar que las cosas existen, y son como el pensamiento se las representa. Por esto el problema gnoseológico es el problema de la filosofía moderna, entendido en esta nueva forma. Ningún pensador moderno rechaza la definición clásica de la verdad (adecuación del intelecto y las cosas), pero ésta se considera como una pura "definición nominal" que no sirve para decir si, y cuando, una afirmación es verdadera. Por lo tanto se buscan criterios de verdad, y éstos se distinguen según dos tipos de posturas. Los filósofos que admiten el presupuesto de una intuición intelectual consideran a las ideas como representaciones de esencias, y confían en poder demostrar su correspondencia con la estructura del mundo de las cosas por medio de un análisis lógico de las representaciones intelectuales (aquí se encuentran, a partir de Descartes, las raíces de la concepción coherentista de la verdad que caracteriza a las corrientes racionalistas del pensamiento moderno y culmina en la filosofía idealista de Hegel y sus continuadores). Estos pensadores no toman en cuenta la observación, ya hecha por Aristóteles, según la cual ningún análisis de la esencia permite afirmar la existencia de cualquier cosa, ya que el juicio de existencia no es reducible a la aplicación de un predicado a un sujeto (esta misma observación será enérgicamente reiterada por Kant). Los filósofos que no reconocen la intuición intelectual concentran todo el conocimiento en la sensación, y atribuyen al intelecto la mera capacidad de conectar, comparar y componer las representaciones proporcionadas por los sentidos (admitiendo a veces un sentido interno a lado del sentido externo) sin por otro lado alcanzar nuevos conocimientos. Ésta es la posición de las corrientes empiristas del pensamiento moderno.

Ambas corrientes compartían, además del presupuesto dualista o dualismo epistemológico ya mencionado, el presupuesto dictado por el sentido común de que existe un mundo de cosas, aunque no directamente accesible a nuestro conocimiento. Éste es el mismo presupuesto de la ciencia natural moderna, el cual no tiene otro sentido que el de ser un esfuerzo para conocer a la naturaleza, y por ello es preciso llamarlo "presupuesto naturalista". Esta situación brindaba una cierta ventaja cultural a las corrientes empiristas, ya que en ellas la confianza de que la sensación nos ponga "en contacto" con las cosas descansaba en el hecho de concebir a la sensación como el efecto de una 
acción causal y física producida por las cosas sobre los órganos materiales de nuestros sentidos. Pero ya Descartes había anotado que no hay ninguna razón para afirmar una similitud entre la sensación y los objetos que la producen (por ejemplo, no hay ninguna similitud entre una pluma y la sensación de cosquilleo que ella produce). La confirmación de la imposibilidad de solucionar el problema de la verdad mediante los instrumentos del empirismo se encuentra en el representante más coherente de esta tradición, es decir, en Hume, el cual no desconoce la legitimidad de las convenciones del sentido común acerca de la existencia de las "cosas" o "sustancias" concretas, y de las relaciones de causalidad entre los acontecimientos, pero la reduce a una "creencia” (belief) que carece de toda justificación racional (según la forma de racionalidad empírica admitida por su filosofía).

\section{Las novedades de la ciencia natural moderna}

Frente a todo esto se presentaba la evidencia histórica de la ciencia natural moderna, la cual se ofrecía como un conocimiento verdadero de la naturaleza, y hasta dotado de aquella universalidad, necesidad y certeza (fundadas en el conocimiento de las leyes naturales) que eran precisamente las características de la ciencia según la tradición. Esta situación impresionó profundamente a Kant, el cual trató de explicar cómo un tal conocimiento científico había sido posible, pero siempre dentro de los tres presupuestos mencionados, es decir: el dualismo epistemológico (conocemos nuestras representaciones y no las cosas en sí mismas), la negación de la intuición intelectual (el intelecto no conoce objetos suyos, y se limita a unificar el conjunto de las impresiones sensibles), el presupuesto naturalista (el mundo de las cosas existe como campo de noúmenos pensables, mas no cognoscibles). Su notoria solución es que una ciencia es posible solamente como conocimiento de fenómenos (que son "simples apariencias" pasivamente recibidas por nuestros sentidos), los cuales son unificados y organizados en un mundo de objetos por la acción del intelecto que les aplica sus formas puras a priori, es decir, las categorías. De tal manera, el conocimiento alcanza también universalidad y necesidad, en cuanto siempre y necesariamente se conoce aplicando dichas condiciones $a$ priori. En cuanto a la verdad, aparte de la verdad de los juicios analíticos, que es únicamente formal, en cuanto consiste en explicitar en el predicado lo que ya está contenido en la significación del sujeto (y entonces no corresponde a ningún incremento de conocimiento), la verdad de un auténtico conocimiento se reduce a la conformidad con las condiciones a priori del conocimiento (es decir, aplicación de las categorías a los datos fenoménicos). En un sentido muy particular, Kant habla también de la posibilidad de una "metafísica 
como ciencia", y la presenta como una reflexión sobre las condiciones a priori del conocimiento y de la moral.

No sería difícil mostrar que la interpretación kantiana de la ciencia natural moderna no es históricamente correcta. En realidad, Galileo, por ejemplo, afirma que nuestro conocimiento de las "sustancias naturales" no puede alcanzar su "esencia intrínseca", pero sí puede alcanzar ciertas "afecciones" o "accidentes" de ellas. La diferencia se refiere a una distinción entre sustancia y accidentes muy normal en la ontología tradicional, en la cual los accidentes no son puras apariencias. Además, Galileo habla de "accidentes reales", y los identifica con las propiedades matematizables de los cuerpos, de manera que un conocimiento matemático de la naturaleza nos lleva a conocer la "verdadera constitución del universo". La intuición intelectual no es descartada, ya que no es gracias a los sentidos que se puede leer matemáticamente el mundo y construir aquellos modelos matemáticos ideales que permiten entender, idealizándolos, los acontecimientos físicos y pueden ser sometidos al control experimental que, una vez más, comporta la realización concreta de condiciones artificiales ideales. El mismo Newton, poniéndose explícitamente en el surco de Galileo, no admite en la física la referencia a "cualidades ocultas" y exige que todo discurso se desarrolle a partir de los "fenómenos". Pero éstos no son "puras apariencias", sino los aspectos "manifiestos" de los acontecimientos naturales, de los cuales resulta el conocimiento científico gracias a "generalizaciones" y matematizaciones que son obra del intelecto. En conclusión, desde su fundación hasta finales del siglo XIX la cultura occidental consideró a la ciencia natural no solamente como la forma más perfecta de conocimiento, sino también como conocimiento verdadero (y por otro lado es difícil estimar cualquier conocimiento si no se le concibe como verdadero).

\section{Las consecuencias de la crisis de las ciencias exactas}

La situación cambió como consecuencia de la crisis que afectó a las ciencias exactas (matemática y física) hacia el final del siglo XIX. Mucho tiempo antes del surgimiento de la relatividad y de la física cuántica, la mecánica (es decir, la mecánica de tipo newtoniano y sus prodigiosos desarrollos ulteriores) se había mostrado incapaz de explicar de manera satisfactoria los fenómenos termodinámicos y electrodinámicos. Cuando a todo esto se añadieron los descubrimientos y teorías de la "nueva física" relativista y cuántica, en la cual conceptos, principios y leyes de la física clásica resultaron profundamente modificados y a veces "falsificados", pareció a muchos inevitable reconocer que dicha física se había mostrado falsa. La cosa alegró a varios filósofos, 
quienes aprovecharon la ocasión para reivindicar a la filosofía como el conocimiento verdadero, descalificando a la ciencia. Pero más interesante es considerar la posición de varios autores que siguieron atribuyendo a la ciencia una posición privilegiada. El ejemplo más típico es el de Ernst Mach. Según su diagnóstico, a pesar de la lucha que los positivistas habían llevado contra la metafísica, ésta se había sutilmente insinuado dentro de la misma ciencia. En efecto, rechazar a la metafísica no significa solamente rechazar toda referencia a principios "suprasensibles", sino también rechazar toda pretensión de captar la "verdadera constitución" de lo real. Pero los científicos y los intelectuales que habían atribuido a la ciencia el privilegio de ser la forma más perfecta de conocimiento habían terminado por considerarla como capaz de brindarnos el conocimiento de la verdadera constitución de la naturaleza. Esto había sido el error fatal, al cual no se escapa ni postulando que hay otras formas de saber que pueden brindarnos este conocimiento, ni tampoco imaginando que sería posible encontrar dentro de la misma ciencia nuevas teorías capaces de descubrir la verdadera constitución del mundo natural. La posición correcta consiste en reconocer que los únicos contenidos de conocimiento son los que nos brindan las percepciones sensoriales (empirismo radical), y que el intelecto no tiene ninguna capacidad ni tarea de conocer, sino únicamente la función de establecer ciertas conexiones entre las percepciones que son "económicamente" útiles para permitirnos algunas previsiones probables acerca de futuras percepciones y, de esta manera, proporcionarnos ciertas indicaciones útiles para nuestra conducta práctica. Por lo tanto, conceptos, leyes, principios, modelos teóricos que constituyen el tejido de las teorías científicas no tienen el sentido y el valor de conocimientos del mundo, sino el de convenciones, que es razonable conservar en tanto nos sirvan para los fines prácticos mencionados, pero que también es razonable abandonar y cambiar por otras cuando ya no sirvan (convencionalismo pragmatista). Tres cosas merecen ser apuntadas: por primera vez en la historia de la cultura occidental se declara abiertamente que la ciencia no alcanza la verdad, además se niega a la ciencia el carácter de ser un conocimiento en sentido propio; todo esto es la consecuencia de haber negado claramente, una vez más, la posibilidad de una intuición intelectual.

\section{La interpretación neopositivista de la ciencia}

Después de un cierto tiempo la confianza en el poder cognoscitivo de la ciencia se recuperó suficientemente y resurgió hasta la persuasión de que ella es la forma más adecuada de conocimiento. Ésta fue la postura del neopositivismo o empirismo lógico, el cual heredó las ideas básicas de Mach, con algunas 
importantes integraciones. El empirismo radical quedó inalterado, así como la negación de la intuición intelectual. A la razón le fue reconocida una función más importante, ya que los neopositivistas se presentaron como campeones de la nueva lógica matemática, la cual, sin embargo, era concebida como un poderoso instrumento para alcanzar el rigor analítico y demostrativo, pero no como instrumento para producir nuevos conocimientos capaces de sobrepasar la base empírica. Además esta corriente se inscribió totalmente dentro del surco del giro lingüístico (linguistic turn) que caracterizó a gran parte de la filosofía contemporánea, lo que llevó a considerar a la ciencia como un conjunto de construcciones lingüísticas (las diferentes teorías científicas), y a ver la filosofía de la ciencia como un análisis lógico-lingüístico de sus teorías. El empirismo radical fue inyectado en el plano lingüístico bajo la forma de un "criterio de significación", expresado por el famoso principio de verificación (el significado de una proposición es el método de su verificación empírica). Con estos instrumentos el neopositivismo reanudó la lucha contra la metafísica, que es el corazón de su programa cultural, apuntado a promover una concepción científica del mundo: sometiendo a un análisis riguroso cualquier discurso metafísico se descubre que, muchas veces, es simplemente incoherente desde el punto de vista lógico; si no es así, es posible mostrar que contiene términos desprovistos de sentido; por lo tanto, el discurso metafísico queda totalmente derrocado.

Aunque no haya profundizado en este tema explícitamente, el neopositivismo aceptaba tácitamente que la ciencia es un saber verdadero, ya que los términos observacionales, que reflejan en su lenguaje la referencia empírica al mundo, permiten afirmar que ella dice la verdad a propósito del mundo. Sin embargo, era claro que el discurso de cualquier ciencia incluye muchos términos teóricos que no admiten una verificación empírica directa. En un primer momento, los empiristas lógicos creyeron que era posible, mediante un trabajo de definiciones lógicas, eliminar en principio los términos teóricos, reduciendo su significación a la de los términos observacionales. Pero, profundizando en la teoría del análisis lingüístico, la filosofía analítica (que es en cierto sentido la heredera del empirismo lógico) llegó a afirmar que la auténtica "unidad de significado" no es ni siquiera la proposición, sino el contexto global del discurso. En el caso de las ciencias este contexto es la teoría científica completa, y, por lo tanto, el significado de todos sus términos depende del contexto teórico, y los mismos términos observacionales no constituyen una excepción a esta regla fundamental. Entonces, en un sentido radical, todos los términos son teóricos (holismo semántico de Quine). Esta conclusión constituía una verdadera "autorrefutación" del empirismo lógico, y además implicaba consecuencias desastrosas para la ciencia misma. En primer lugar, resultaba imposible comparar las teorías científicas con base en su 
compatibilidad con los datos empíricos, porque éstos no son los mismos datos para una y para otra. Por consiguiente, no se podía tampoco decir si una teoría es "mejor" que otra, y, por tanto, no resultaba posible hablar de progreso en la ciencia. Vino desarrollándose así una serie de filosofías de la ciencia conocidas como postempiristas, en las cuales se subrayaron con fuerza los contextos sociales e históricos que rodean el quehacer de la investigación científica y de sus aplicaciones. El constituirse, afirmarse y desaparecer de teorías científicas fue interpretado como el efecto de varios factores, entre los cuales los criterios de la tradicional racionalidad científica (es decir, la fuerza de la evidencia empírica y del rigor lógico) tienen un papel muy marginal. No podemos detenernos en detalles acerca de este cuadro bastante bien conocido de la epistemología actual. Lo que nos interesa notar es que la empresa científica se coloca en un plano esencialmente pragmático, y la noción de verdad desaparece de la ciencia, siendo sustituida por la noción de aceptación, la cual puede ser determinada por un número muy amplio e indeterminado de factores contingentes. En ciertos autores amantes de la paradoja, como Feyerabend, se llega incluso a afirmar que no hay superioridad de las ciencias sobre las pseudociencias, como la astrología o la magia.

\section{Una revisión del concepto de verdad científica}

El problema que nos interesa discutir ahora es si esta profunda crisis de la verdad científica, la cual ha contribuido poderosamente a alimentar la desconfianza en la verdad que está permeando nuestra civilización, es justificada, o si ella no depende, más bien, de ciertas equivocaciones básicas que afectaron la visión de la ciencia que afirmaban sus supuestos paladines, es decir, los filósofos de inspiración neopositivista. Estamos convencidos que así es y, para mostrarlo, pasamos a considerar una de estas equivocaciones fundamentales.

En primer lugar, no es correcto reducir las teorías científicas a constructos lingüísticos (linguistic turn) en los cuales todo lo que cuenta son las conexiones lógicas entre enunciados. Desde este punto de vista resulta normal considerar una teoría como la conjunción lógica de todas sus hipótesis. Si fuera así, el descubrimiento de una sola consecuencia lógica falsa (es decir, incompatible con un único resultado experimental) sería suficiente para declarar falsa la teoría entera. Así pensaba Popper, mas el hecho concreto de que nunca se abandona una teoría como consecuencia de una sola desmentida (y hasta de varias desmentidas), produjo correcciones del tipo del "falsificacionismo sofisticado" de Lakatos, de la doctrina de los paradigmas de Kuhn, de la concepción "estructuralista" de Sneed-Stegmüller, y otras propuestas. 
Éstas son interesantes, pero no captan el punto esencial que podemos esbozar del modo siguiente. Una cierta ciencia se instituye cuando el mundo de las cosas es considerado únicamente desde el punto de vista de algunos atributos específicos que resultan de una abstracción intelectual. Para entender y explicar los acontecimientos observables en los cuales ocurren estos atributos, el científico elabora un cuadro intelectual global o modelo intelectual de su campo de investigación, en el cual varios elementos teóricos parecen ofrecer una explicación causal de los acontecimientos observados. Este momento hermenéutico preliminar tiene que "explicitarse", y esta explicitación se realiza de dos maneras: el contenido intuitivo del modelo se articula en un número finito de hipótesis y se determinan ciertos procedimientos operacionales concretos para asegurar la referencia empírica controlable a los atributos seleccionados. Resulta así la teoría, la cual es una cierta descripción lingüística del modelo intuitivo, y en ella la explicación causal toma la forma de la deducción lógica. En esta perspectiva no todas las hipótesis tienen el mismo peso y la misma importancia, y esto permite, en el caso de que un cierto resultado experimental sea incompatible con una hipótesis, modificarla sin abandonar la teoría, siempre y cuando esta hipótesis no ocupe un "lugar central" (en sentido intelectual) en el modelo. Si, por lo contrario, el resultado experimental se opone directamente a uno o más de los elementos fundamentales del modelo intelectual, éste entra en crisis y la teoría que lo explicita tiene que ser abandonada.

Hablando de la función referencial de los procedimientos operacionales hemos tocado otro punto importante. La teoría contextual u holística del significado oculta una distinción bien conocida al pensamiento clásico y también subrayada por Frege en la época moderna: la distinción entre sentido y referencia. El sentido es algo mental, intelectual, mientras que la referencia es algo extralingüístico y extramental. Por lo tanto, es correcto afirmar que el sentido de un concepto depende (en gran parte, por los menos) de la red conceptual en la cual se encuentra, y que eso puede ser esclarecido pensando y hablando, pero el referente, el objeto concreto, no se alcanza ni pensando ni hablando, sino actuando. Por lo tanto, aunque el significado completo de un concepto o término operacional dependa también de su contexto teórico, hay en ello un núcleo referencial directamente ligado a las operaciones que permite delimitar el campo de objetos de una teoría y es independiente de la teoría misma. Por consiguiente, si dos teorías admiten los mismos procedimientos operacionales de referencia, resultan comparables si se puede determinar un experimento totalmente operacional cuyo resultado sería compatible con una e incompatible con la otra. Es claro que, si esta condición no se da, dichas teorías serán parcialmente o totalmente incomparables y esto signifi$\mathrm{ca}$, en último análisis, que ellas se refieren a objetos diferentes. 
Después de lo dicho es posible retomar la noción de verdad en la ciencia, sin reducirla a su forma débil de pura coherencia interna, sino tomándola en su sentido fuerte de adecuación entre el pensamiento -o el discurso que lo expresa- y un mundo extramental y extralingüístico. En su sentido más preciso, la verdad es una propiedad relacional que se atribuye a una proposición si ésta está en una relación correcta con aquello a lo que se refiere. Por lo tanto, un discurso nunca es verdadero o falso en sí mismo, sino en la medida en que dice o no dice de sus referentes lo que éstos realmente son. Habiendo recuperado la dimensión referencial de la ciencia, es normal que se pueda también recuperar por ella la dimensión de la verdad. Sin embargo, hay que subrayar que la verdad siempre es relativa al campo de referencia del discurso. Cuando al principio de nuestro siglo se pensó que las teorías de la física clásica se habían revelado "falsas", se concebía la verdad como algo absoluto, es decir, como si la física tuviera que ser verdadera a propósito de la realidad física en sentido total. Por consiguiente, si acontece que ciertos aspectos de dicha realidad contradicen a una teoría física, ésta tiene que ser falsa. Sin embargo, hemos visto que ninguna ciencia hace objeto de su investigación a la realidad total (ni siquiera un subdominio completo de la realidad), sino únicamente a ciertos objetos determinados como conjunto estructurado de pocos atributos a los cuales se refiere gracias a bien determinados procedimientos operacionales. Es posible, entonces, afirmar que la física clásica era verdadera (y sigue siendo verdadera) sobre sus objetos, y que esta verdad no es incompatible con la de otras teorías físicas que se ocupan de nuevos objetos (en el sentido exacto precisado arriba), los cuales son determinados y resultan accesibles gracias a procedimientos operacionales diferentes (a pesar del hecho, también importante, que los mismos conceptos reciben una interdefinición "teórica" a veces muy diferente).

Hay que decir que la noción de verdad aquí utilizada se aplica directamente a las proposiciones aisladas, y que su aplicación a las teorías necesita de muchas precisiones ya que, como se sabe, cada teoría es "subdeterminada" con respecto a la evidencia empírica de la cual se ocupa. Pero la sustancia del discurso no cambia: en la medida que, utilizando varios criterios epistémicos de los cuales trata la filosofía de la ciencia, resulta razonable dar la preferencia a una cierta teoría, esto significa que es razonable considerarla verdadera.

Aparentemente estamos admitiendo algo que parece destruir la noción misma de verdad, ya que admitimos que no hay criterios absolutamente seguros para afirmarla. Pero esta objeción confunde verdad y certeza. La verdad es una característica objetiva de una proposición y la certeza es una característica subjetiva que concierne a nuestra posesión de la verdad. Por lo tanto, así como la existencia de la verdad no implica la imposibilidad del error, del mismo modo nuestra posibilidad de alcanzar la verdad no implica que siem- 
pre estamos ciertos de haberla alcanzado y de no haber incurrido en errores. Esto vale en general (es decir, en todos los ámbitos de la vida humana) y se aplica también a la ciencia. Las certezas humanas siempre son "prácticas" en este sentido: que no hay duda razonable para no admitirlas. Lo mismo vale para la ciencia: tenemos el derecho de considerarla como la fuente de muchísimos descubrimientos y teorías que han dilatado inmensamente nuestro saber, proporcionándonos un conocimiento fiable en muchos sectores parciales. La idea de la infalibilidad de la ciencia pertenece al pasado de un cientificismo positivista históricamente muerto, pero la idea de un conocimiento falible más capaz de alcanzar verdades que se pueden aceptar más allá de toda duda razonable corresponde a una apreciación correcta de lo que la ciencia merece a los ojos del hombre actual. 